\title{
Genetic structure of Brazilian wild rice (Oryza glumaepatula Steud., Poaceae) populations analyzed using microsatellite markers
}

\author{
Marines M.G. Karasawa ${ }^{1}$, Roland Vencovsky ${ }^{1}$, Cynthia M. Silva ${ }^{1}$, Maria Imaculada Zucchi ${ }^{2}$, \\ Giancarlo C.X. Oliveira ${ }^{1}$ and Elizabeth A. Veasey ${ }^{1}$ \\ 'Departamento de Genética, Escola Superior de Agricultura "Luiz de Queiroz", \\ Universidade de São Paulo, Piracicaba, SP, Brazil. \\ ${ }^{2}$ Instituto Agronômico de Campinas, Campinas, SP, Brazil.
}

\begin{abstract}
Knowledge of the genetic structure and diversity of natural populations is important in developing strategies for in situ and ex situ conservation. We used eight microsatellite loci to estimate genetic structure and investigate within and between population genetic variation in eleven Brazilian wild rice (Oryza glumaepatula) populations. The study showed the following genetic diversity parameters: average number of 3.1 alleles per locus; $77.3 \%$ polymorphic loci; 0.091 observed heterozygosity and 0.393 gene diversity. F-statistics detected by microsatellite loci were: $F_{S T}=0.491$ (and $\left.R_{S T}=0.608\right), F_{I S}=0.780$ and $F_{I T}=0.888$. No population was in Hardy-Weinberg equilibrium. The estimated apparent outcrossing rate $\left(\bar{t}_{a}=0.143\right)$ indicated a predominance of self-fertilization. The gene flow values were low ( $N m=0.259$ and 0.161 for $F_{S T}$ and $R_{S T}$, respectively). Populations were spatially structured but without a correlation between genetic and geographic distances. Five populations (PG-4, PG-2, PU-1, SO-4, NE-18) were identified as priorities for conservation strategies. Populations from the Amazon biome showed heterogeneity with respect to intrapopulation diversity $\left(\bar{H}_{e}\right)$. The high level of genetic differentiation between populations and the high number of private alleles suggested that sampling should be carried out on a large number of $O$. glumaepatula populations for ex situ conservation purposes.
\end{abstract}

Key words: conservation, gene flow, genetic diversity, microsatellites, Oryza glumaepatula.

Received: March 9, 2006; Accepted: October 3, 2006.

\section{Introduction}

There are 23 species in the rice genus Oryza, one of which is the wild rice Oryza glumaepatula Steud. (Poaceae) which has an AA genome, of the same type as the cultivated Oryza sativa, and occurs widely in Latin America from $23^{\circ} \mathrm{N}$ in Cuba to $23^{\circ} \mathrm{S}$ in Brazil (Vaughan et al., 2003). The Brazilian O. glumaepatula populations are found in the extensive river basins of the Amazon and Pantanal Matogrossense and the smaller river basins which occur in the states of Goiás and Tocantins (Oliveira, 1994; Brondani et al., 2005).

Several studies have indicated that $O$. glumaepatula is a self-fertilizing species (Akimoto et al., 1998; Buso et al., 1998; Ge et al., 1999). Such species are usually more homogeneous within populations but more differentiated between populations, forming pure lines within families and increasing the genetic differentiation between families

Send correspondence to EA Veasey, Departamento de Genética, Escola Superior de Agricultura "Luiz de Queiroz", Universidade de São Paulo, Caixa Postal 83, 13400-970 Piracicaba, SP, Brazil. E-mail: eaveasey@esalq.usp.br. by generating subdivision within the population. The fixation of specific alleles may confer little or no flexibility in response to environmental changes and, in extreme cases, these species may become completely extinct due to allele fixation and loss of genetic diversity (Richards, 1997; Hedrick, 2001). In favorable environments such species may exhibit aggressive behavior typical of weeds and colonizing plants and rapidly become established (Holsinger, 2000). These types of species need special attention from conservationists and the highest possible number of populations should be identified to increase the chance of conserving different alleles and thus preserve the diversity existing within the species with higher efficiency. To develop strategies for in situ and ex situ germplasm conservation and plan future plant collections it is, therefore, important to gather information on the genetic variation within and between natural populations and the reproductive biology of these populations.

Molecular markers have been used for germplasm characterization, phylogenetic and population genetic studies in O. glumaepatula and several other Oryza species 
(Akimoto et al., 1998; Buso et al., 1998; Ge et al., 1999; Doi et al., 2000; Bautista et al., 2001; Oliveira, 2002; Gao et al., 2002a, 2002b; Gao, 2004; Brondani et al., 2005). Buso et al. (1998) used isozyme and random amplified polymorphic DNA (RAPD) markers to estimate genetic parameters for four South American O. glumaepatula populations and found high values for interpopulation differentiation.

Doi et al. (1996) analyzed accessions of AA genome Oryza species and concluded that $O$. glumaepatula is a subtype of Oryza rufipogon Griff. In a later study, Ge et al. (1999) used RAPD markers to investigate natural Chinese and Brazilian O. rufipogon populations and found that Chinese populations were more polymorphic at both the population and regional levels, thus concluding that the high degree of genetic differentiation between Chinese and Brazilian populations agrees with the classification of the Latin American form of $O$. rufipogon as the separate species $O$. glumaepatula. More recently, Buso et al. (2001) used RAPD and specific sequences of nuclear and cytoplasmatic DNA coupled with phenetic and cladistic analyses to produce phylogenetic trees verifying that $O$. glumaepatula was a separate species distinct from $O$. rufipogon. Doi et al. (2000) studied the variation in the mitochondrial, chloroplast and nuclear DNA of O. glumaepatula and suggested that this species had multiple origins, while Bautista et al. (2001) investigated the phylogenetic relationships between cultivated and wild rice species and concluded that $O$. glumaepatula was relatively close to the cultivated rice species $O$. sativa and Oryza glaberrima.

Microsatellites have been widely used in studies of population genetics of wild plants due to their reproducibility and the fact that they are more genetically informative than is the case for other markers (Ferreira and Grattapaglia, 1998), especially in populations with low genetic diversity at the DNA level (Peatkau et al., 1995). Brondani et al. (2005) used ten microsatellite markers to analyze 30
O. glumaepatula populations from the Brazilian Amazon, Cerrado and Pantanal biomes, assessing six to 24 plants per population, and found high genetic diversity between populations but high inbreeding levels within populations, suggesting a preferentially self-pollinating breeding system.

We used eight microsatellite markers to estimate gene flow and genetic structure and analyze within and between population genetic variation in eleven Brazilian $O$. glumaepatula populations and to address specific strategies for the in situ and ex situ conservation of this species. This is a complementary study to previous studies of $O$. glumaepatula using microsatellite markers with a larger sample of populations from the Amazon biome plus one particular population from the Xingu river in the Brazilian state of Mato Grosso, performing spatial correlation analysis and sampling a higher number of plants per population.

\section{Material and Methods}

\section{Plant material}

We investigated eleven Brazilian Oryza glumaepatula Steud. (Poaceae) populations held at the wild rice germplasm collection of the Genetics Department of Escola Superior de Agricultura "Luiz de Queiroz" (ESALQ/USP), Piracicaba, SP, Brazil. Seven of the populations (JA-4, NE-18, NE-26, PU-1, SO-4, SO-6 and TA-1) were from the basins of rivers tributary to the Amazon in the northern Brazilian states of Roraima and Amazonas between $01^{\circ} 53 \mathrm{~S}$ and $03^{\circ} 49 \mathrm{~S}$ in the Amazon biome, one population (XI-1) was also from an Amazon tributary but from the more southern state of Mato Grosso at $12^{\circ} 14 \mathrm{~S}$ in the Amazon biome, and three populations (PG-1, PG-2 and PG-4) were from the Paraguay river basin even further south in the state of Mato Grosso do Sul at between $19^{\circ} 00 \mathrm{~S}$ and $19^{\circ} 15 \mathrm{~S}$ in the Pantanal biome (Table 1, Figure 1). The population codes refer to the river basins, not the rivers where the plants were isolated.

Table 1 - Origin of the 11 Brazilian Oryza glumaepatula populations evaluated. The populations are ordered by latitude, starting with the most equatorial. Except for the XI-1 population, which was collected from a River Amazon tributary in Mato Grosso state, all the Amazon biome populations were collected from River Amazon tributaries in Amazonas and Roraima states. The Pantanal biome populations were collected in Mato Grosso do Sul state.

\begin{tabular}{|c|c|c|c|c|c|}
\hline $\begin{array}{l}\text { Biome and } \\
\text { population code }\end{array}$ & $\begin{array}{l}\text { Sample size } \\
\text { (n) }\end{array}$ & River basin & River & Lake & Map reference \\
\hline \multicolumn{6}{|l|}{ Amazon biome } \\
\hline NE-18 & 30 & Negro & Branco & - & $01^{\circ} 53 \mathrm{~S}, 61^{\circ} 22 \mathrm{~W}$ \\
\hline NE-26 & 22 & Negro & Carabinani & - & $01^{\circ} 54 \mathrm{~S}, 61^{\circ} 23 \mathrm{~W}$ \\
\hline $\mathrm{JA}-4$ & 36 & Japurá & Japurá & Cuiucuiú & $02^{\circ} 02 \mathrm{~S}, 65^{\circ} 07 \mathrm{~W}$ \\
\hline TA-1 & 36 & Tapajós & Tapajós & - & $02^{\circ} 26 \mathrm{~S}, 54^{\circ} 42 \mathrm{~W}$ \\
\hline SO-6 & 18 & Solimões & Solimões & Manacapuru & $03^{\circ} 11 \mathrm{~S}, 60^{\circ} 47 \mathrm{~W}$ \\
\hline $\mathrm{SO}-4$ & 30 & Solimões & Solimões & Calado & $03^{\circ} 16 \mathrm{~S}, 60^{\circ} 13 \mathrm{~W}$ \\
\hline PU-1 & 30 & Purus & Purus & - & $03^{\circ} 49 \mathrm{~S}, 61^{\circ} 25 \mathrm{~W}$ \\
\hline XI-1 & 30 & Xingu & Xingu & Piulaga & $12^{\circ} 14 \mathrm{~S}, 53^{\circ} 35 \mathrm{~W}$ \\
\hline \multicolumn{6}{|l|}{ Pantanal biome } \\
\hline PG-2 & 18 & Paraguai & Paraguai & - & $19^{\circ} 00 \mathrm{~S}, 57^{\circ} 41 \mathrm{~W}$ \\
\hline PG-1 & 30 & Paraguai & Paraguai & - & $19^{\circ} 01 \mathrm{~S}, 57^{\circ} 30 \mathrm{~W}$ \\
\hline PG-4 & 30 & Paraguai & Taquari & - & $19^{\circ} 15 \mathrm{~S}, 57^{\circ} 13 \mathrm{~W}$ \\
\hline
\end{tabular}




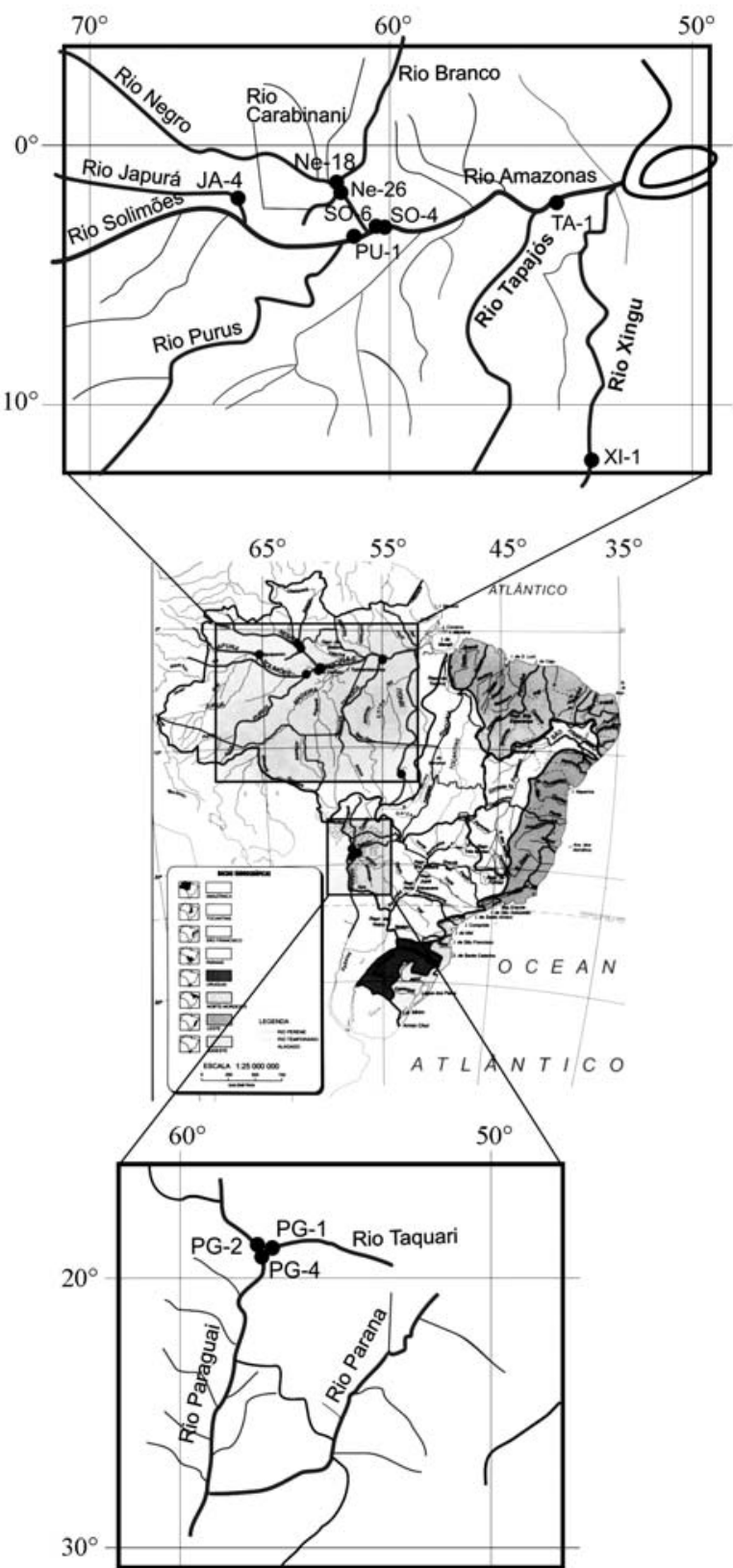

Figure 1 - Map showing the distribution of the 11 Brazilian Oryza glumaepatula populations.

For each population, two to three seeds were sampled from individual plants collected in nature to form a bulk sample of 50 seeds which were germinated in germination chambers at $27{ }^{\circ} \mathrm{C}$ in the dark. Germinated seeds were transplanted to pots containing fertilized soil and cultivated to the adult stage in a greenhouse under natural lighting conditions. The number of adult plants sampled from each population ranged from 18 to 36 (Table 1).

\section{Microsatellite analysis}

Total genomic DNA was extracted from adult lyophilized leaves using a cetyltrimethylammonium bromide
(CTAB) procedure (Hoisington et al., 1994) as simplified by Karasawa (2005) by removing the sodium acetate and all following steps. The DNA was quantified in a $4 \%(\mathrm{w} / \mathrm{v})$ polyacrylamide gel using a silver staining procedure (Bassan et al., 1991).

Eight primer pairs (Table 2) developed by Brondani et al. (2001) for O. glumaepatula were selected for amplification. All primers were first submitted to a basic PCR procedure with $56{ }^{\circ} \mathrm{C}$ for primer annealing (Brondani et al., 2001). Primers that did not amplify satisfactorily were submitted to new amplification cycles with higher and lower annealing temperatures. The primer pairs OG-26 and OG29 amplified satisfactorily at $60{ }^{\circ} \mathrm{C}$, and OG-27 amplified at $54{ }^{\circ} \mathrm{C}$.

For each PCR reaction, $30 \mathrm{ng}$ of genomic DNA were used in a $12 \mu \mathrm{L}$ volume containing $0.3 \mu \mathrm{M}$ of each primer, $0.25 \mathrm{mM}$ of each dNTP, $1.5 \mathrm{mM}$ of $\mathrm{MgCl}_{2}, 10 \mathrm{mM}$ Tris$\mathrm{HCl}$, and 0.6 unit of Taq DNA polymerase enzyme (Gibco $\mathrm{BRL}$ ). The reactions were performed on a Primus 96 Thermocycler with 4 min initial denaturation at $94^{\circ} \mathrm{C}, 30$ subsequent cycles $\left(1 \mathrm{~min}\right.$ at $94^{\circ} \mathrm{C}, 1 \mathrm{~min}$ at $54^{\circ} \mathrm{C}, 56^{\circ} \mathrm{C}$ or $60^{\circ} \mathrm{C}$, 1 min elongation at $72^{\circ} \mathrm{C}$ ), followed by a final elongation of $5 \mathrm{~min}$ at $72^{\circ} \mathrm{C}$. Amplified products were electrophoresed on $6 \%$ non-denaturing polyacrylamide gels run vertically ( $120 \mathrm{~V}$ for $3 \mathrm{~h}$ ). Amplified fragments were visualized using a silver staining procedure (Bassan et al., 1991) (Figure 2).

\section{Data analysis}

Genotypic frequencies were submitted to Fisher's exact test considering Hardy-Weinberg equilibrium proportions (Weir, 1996), using the TFPGA program (Miller, 1997). Allelic and genotypic frequencies, mean number of alleles per locus $(\bar{A})$, average percentage of polymorphic loci $(\bar{P})$, mean observed heterozygosities $\left(\bar{H}_{o}\right)$, gene diversity $\left(\bar{H}_{e}\right)$, and Wrights $\mathrm{F}$ statistics were estimated using the GDA program (Lewis and Zaykin, 2000). Private alleles were also identified under the GDA program (Lewis and Zaykin, 2000).

The mutation process in microsatellite loci is not in line with the expectations under the infinite alleles model with low rates. Therefore, the analogue of the $F_{S T}$ parameter (Slatkin, 1995) developed specifically for microsatellite data $\left(R_{S T}\right)$ was also estimated. Parameters $R_{S T}$ and gene flow $(\mathrm{Nm})$ were estimated using the $\mathrm{R}_{\mathrm{ST}} \mathrm{Cal}$ program (Goodman, 1997). Dendrograms were constructed from Nei's genetic distances matrix and the unweighted pair group method with averages (UPGMA) clustering criteria using the TFPGA program (Miller, 1997).

Spatial variation patterns were analyzed using Pearson's coefficient of correlation (r) between Nei's genetic distances matrix (Nei, 1978) and the matrix of geographic distances (shortest distance between two given points on the map) between populations, using the NTSYS-pc program (Rohlf, 1992). Significance of these correlations was tested using Mantel's Z statistic (Mantel, 1967) and 1000 
Table 2 - Simple sequence repeat (SSR) loci, primer-pair sequences developed for Oryza glumaepatula (Brondani et al. 2001), chromosome number $(\mathrm{CN})$, allele size range in base pairs (bp), number of alleles per locus $(\mathrm{A})$ and annealing temperatures $\left(T_{a}\right)$. The mean number of alleles per locus (A) was 10 .

\begin{tabular}{|c|c|c|c|c|c|}
\hline SSR loci & Primer sequences & $\mathrm{CN}$ & $\mathrm{bp}$ & $\mathrm{A}$ & $T_{a}\left({ }^{\circ} \mathrm{C}\right)$ \\
\hline \multirow[t]{2}{*}{ OG 22} & (F) GCCATCCATTCTTACCAG & 12 & $165-265$ & 20 & 56 \\
\hline & (R) CACAGGTGTGGTGCTCA & & & & \\
\hline \multirow[t]{2}{*}{ OG 26} & (F) CATGGTGCCGATTACGGT & 10 & $95-120$ & 5 & 60 \\
\hline & (R) CATCTCCATCGCGGTCAT & & & & \\
\hline \multirow[t]{2}{*}{ OG 27} & (F) TCGGACGTGGCATATGA & 9 & $120-210$ & 16 & 54 \\
\hline & (R) CTGTTCCGAGCGAGAGT & & & & \\
\hline \multirow[t]{2}{*}{ OG 29} & (F) GACCAGTTCACCATGCAG & 1 & $80-125$ & 10 & 60 \\
\hline & (R) GAGTGAGGCAGCAAGACA & & & & \\
\hline \multirow[t]{2}{*}{ OG 36} & (F) AACGTTCATCGGTTCTGG & 4 & $150-190$ & 8 & 56 \\
\hline & (R) TGCTTGCCAGGTTATTCC & & & & \\
\hline \multirow[t]{2}{*}{ OG 39} & (F) GCGTACTAGGCCATGATA & 3 & $250-275$ & 6 & 56 \\
\hline & (R) TCCACGTAAGAACACTCG & & & & \\
\hline \multirow[t]{2}{*}{ OG 42} & (F) TGCAGGCTCTGAGCTAC & 5 & $420-440$ & 6 & 56 \\
\hline & (R) AGAACAGATCTTGCCGTC & & & & \\
\hline \multirow[t]{2}{*}{ OG 63} & (F) CAGGGGACAAGCACATA & 2 & $100-150$ & 9 & 56 \\
\hline & (R) TAGACGATGTCGAGAAGG & & & & \\
\hline
\end{tabular}
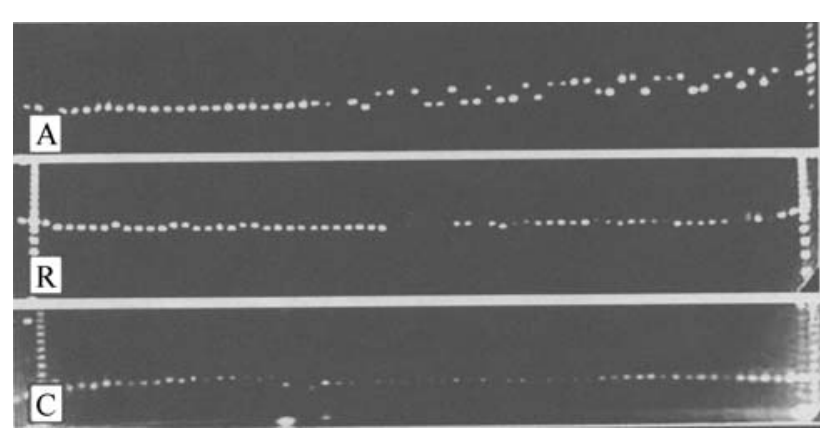

Figure 2 - Non-denaturing polyacrylamide gels of Oryza glumaepatula populations, in the following order: (A) primer OG-26, pop. PU-1 (left) and SO-4 (right); (B) primer OG-26, pop. NE-18 (left) and TA-1 (right), (C) primer OG-29, pop. PU-1 (left) and SO-4 (right). Ladders (10 bp and $100 \mathrm{bp}$ ) are located at the left and right sides of the gels and in the middle of gels A and C.

random permutations. The average apparent outcrossing rate was estimated from the relationship $\bar{t}_{a}=\left(1-F_{I S}\right) /$ $\left(1+F_{I S}\right)$, which is valid only if populations are in equilibrium with respect to the mating system. The parameter $t_{a}$ was also estimated for each population as $t_{a}($ mean $)=\sum_{i}\left[\left(1-f_{i}\right) /(1+f)\right] / n$.

\section{Results}

The eight pairs of primers amplified a total of 80 alleles in 310 plants from the 11 populations of $O$. glumaepatula investigated (Table 2). All loci showed polymorphism over the populations. The number of alleles per locus varied from 5 to 20 with an average of 10 alleles obtained in the 11 populations, although there was considerable variation among populations (Table 3 ). For example, while the River Japurá lake Cuiucuiú JA-4 population was monomorphic for the eight primers and the Carabinani River NE-26 population was also highly monomorphic with only one polymorphic locus (OG-36) having two alleles, in the River Purus PU-1 population we detected 11 alleles at the OG-22 locus. Considering all loci, 20\% were monomorphic in the 11 populations investigated. Populations from the Amazon River basin showed the highest level of monomorphism (30\%), while the Paraguay River basin showed only $4 \%$. These results are not in agreement with the RAPD data obtained by Buso et al. (1998) where monomorphism was higher in plants from the Paraguay region $(32 \%)$ and lower in those of the Amazon region (16\%).

Lower observed heterozygosity values were found for the 11 populations when compared to gene diversity values (Table 4), indicating an excess of homozygotes. Deviations from Hardy-Weinberg equilibrium were significant for all populations. The population from the Xingu River (XI-1) is clearly differentiated, with the highest level of observed heterozygosity $\left(\bar{H}_{o}=0.233\right)$, the lowest coefficient of inbreeding $(f=0.371)$ and the highest apparent outcrossing rate $\left(t_{a}=0.459\right)$. In general, populations were not homogeneous in relation to gene diversity, with $H_{e}$ values ranging from 0.0 to 0.585 , with the highest levels found in plants from the Paraguay River basin (PG-1, PG-4, PG-2), followed by populations SO-4 and NE-18 from the Solimões and Negro river basins, respectively, in the Amazon biome. Regarding the mating system, inferred here through 
Table 3 - Number of alleles per locus for eleven Brazilian Oryza glumaepatula populations.

\begin{tabular}{lcccccccc}
\hline \multirow{2}{*}{$\begin{array}{l}\text { Biome and } \\
\text { population codes }\end{array}$} & \multicolumn{7}{c}{ Number of alleles per locus } \\
\cline { 2 - 8 } Amazon biome & OG-22 & OG-26 & OG-27 & OG-29 & OG-36 & OG-39 & OG-42 & OG-63 \\
\hline NE-18 & 2 & 3 & 4 & 5 & 4 & 2 & 2 & 3 \\
NE-26 & 1 & 1 & 1 & 1 & 2 & 1 & 1 & 1 \\
JA-4 & 1 & 1 & 1 & 1 & 1 & 1 & 1 & 1 \\
TA-1 & 5 & 2 & 6 & 4 & 5 & 3 & 2 & 3 \\
SO-6 & 2 & 1 & 5 & 4 & 1 & 2 & 2 & 3 \\
SO-4 & 3 & 3 & 6 & 4 & 4 & 4 & 2 & 2 \\
PU-1 & 11 & 2 & 4 & 5 & 4 & 3 & 1 & 2 \\
XI-1 & 3 & 2 & 5 & 2 & 2 & 1 & 2 & 3 \\
\hline Pantanal biome & & & & & & & & \\
PG-2 & 5 & 2 & 8 & 6 & 3 & 1 & 3 & 4 \\
PG-1 & 4 & 2 & 7 & 5 & 4 & 2 & 2 & 5 \\
PG-4 & 3 & 2 & 7 & 6 & 4 & 3 & 2 & 7 \\
\hline
\end{tabular}

Table 4 - Average estimates of the genetic parameters based on eight microsatellite loci for eleven Brazilian Oryza glumaepatula populations: number of plants sampled per locus $(\mathrm{N})$, average number of alleles per locus (A), percentage of polymorphic loci (p), observed heterozygosity across loci $\left(\bar{H}_{o}\right)$, gene diversity $\left(\bar{H}_{e}\right)$, fixation index $(f)$ and apparent crossing rate $\left(t_{a}\right)$.

\begin{tabular}{|c|c|c|c|c|c|c|c|}
\hline \multirow{2}{*}{$\begin{array}{l}\text { Biome and } \\
\text { population codes }\end{array}$} & \multicolumn{7}{|c|}{ Genetic parameters } \\
\hline & $\mathrm{N}$ & A & $\mathrm{p}$ & $\bar{H}_{o}$ & $\bar{H}_{e}$ & $f$ & $t_{a}$ \\
\hline \multicolumn{8}{|l|}{ Amazon biome } \\
\hline NE-18 & 29.1 & 3.250 & 100.0 & 0.073 & 0.526 & 0.863 & 0.074 \\
\hline NE-26 & 21.6 & 1.125 & 12.5 & 0.011 & 0.030 & 0.628 & 0.229 \\
\hline JA-4 & 35.4 & 1.000 & 0.0 & 0.000 & 0.000 & - & \\
\hline TA-1 & 32.4 & 3.875 & 100.0 & 0.018 & 0.464 & 0.961 & 0.020 \\
\hline SO-6 & 16.8 & 2.500 & 75.0 & 0.136 & 0.364 & 0.634 & 0.224 \\
\hline $\mathrm{SO}-4$ & 29.6 & 3.500 & 100.0 & 0.054 & 0.527 & 0.898 & 0.054 \\
\hline PU-1 & 29.8 & 4.000 & 87.5 & 0.071 & 0.328 & 0.787 & 0.119 \\
\hline XI-1 & 30.0 & 2.500 & 87.5 & 0.233 & 0.369 & 0.371 & 0.459 \\
\hline \multicolumn{8}{|l|}{ Pantanal biome } \\
\hline PG-2 & 16.6 & 4.000 & 87.5 & 0.126 & 0.562 & 0.780 & 0.123 \\
\hline PG-1 & 30.0 & 3.875 & 100.0 & 0.100 & 0.569 & 0.827 & 0.095 \\
\hline PG-4 & 29.9 & 4.375 & 100.0 & 0.176 & 0.585 & 0.703 & 0.174 \\
\hline Mean & 27.38 & 3.091 & 77.3 & 0.091 & 0.393 & 0.750 & 0.143 \\
\hline
\end{tabular}

the average apparent outcrossing rate $\left(\bar{t}_{a}=0.143\right)$ and the population-level outcrossing rates $\left(t_{a}\right)$, a strong tendency to self-fertilization with a small amount of outcrossing prevailed, with some degree of variation between populations. The exception was population XI-1 that was intermediate, with $45.9 \%$ of outcrossing. The average apparent outcrossing rate is in agreement with the estimate obtained by Brondani et al. (2005) for O. glumaepatula, namely $\bar{t}_{a}=0.135$. The low $\bar{t}_{a}$ estimate found in our study is a consequence of the high intrapopulation coefficient of inbreeding detected (average $f=0.750$, Table 4 ).
The genetic differentiation between populations, relative to the total genetic variation, was very high ( $F_{S T}=0.491$ and $R_{S T}=0.608$ ), these estimates being statistically similar and different from zero (Table 5). Based on the $R_{S T}$ value, the apparent number of migrants per generation was $N m=0.161$, which was a function of the high differentiation between populations. The very high total inbreeding coefficient $\left(F_{I T}=0.888\right)$ was primarily caused by the intrapopulation inbreeding $\left(F_{I S}=0.780\right)$ but differentiation also played an important role in explaining this $F_{I T}$ estimate. 
Table 5 - Estimates of Wright's F statistics, $R_{S T}$ and the number of migrants per generation $\left(N_{m}\right)$ for eleven natural Brazilian Oryza glumaepatula populations.

\begin{tabular}{lcccccc}
\hline & $F_{I T}$ & $F_{S T}$ & $F_{I S}$ & $R_{S T}$ & $N_{m A}{ }^{1}$ & $N_{m B}{ }^{2}$ \\
\hline Under all the loci & 0.888 & 0.491 & 0.780 & 0.608 & 0.161 & 0.259 \\
Upper $\left(\mathrm{CI}_{95 \%}\right)^{3}$ & 0.925 & 0.541 & 0.836 & 0.668 & - & - \\
Lower $\left(\mathrm{CI}_{95 \%}\right)^{3}$ & 0.841 & 0.443 & 0.715 & 0.547 & - & - \\
\hline
\end{tabular}

${ }^{1}$ Based on $R_{S T .}{ }^{2}$ Based on $F_{S T} .{ }^{3} \mathrm{CI}_{95 \%}=95 \%$ confidence interval.

Nei's genetic distances, calculated pairwise between populations, ranged from 0.078 to 0.760 (Table 6). A higher degree of similarity was only found between populations PG-1 and PG-4 and between SO-4 and PU-1. These results, plus the UPGMA dendrogram (Figure 3), indicate that populations from the same regions did not form clusters based on proximity, a conclusion supported by the low correlation between the genetic and geographic distances $(r=0.03)$. Figure 4 shows that private alleles were obtained in nine of the 11 populations reaching relatively large numbers, such as populations PU-1 with eight alleles, PG-2 with six and SO-6 with five alleles. These numbers are significant considering that the mean number of alleles ranged from 1.0 to 4.4 within populations (Table 4 ).

\section{Discussion}

\section{Genetic variation}

High average genetic diversity indices where found for the Brazilian O. glumaepatula populations in this study using microsatellite markers $(\bar{A}=3.091 ; \bar{P}=77.3 \%$; $\left.\bar{H}_{e}=0.393\right)$, in spite of the predominantly self-fertilization mating system of this species. However, the populations were not homogeneous with respect to observed heterozygosities $\left(\bar{H}_{o}\right)$, gene diversity $\left(\bar{H}_{e}\right)$, number of private alleles and apparent outcrossing rates $\left(t_{a}\right)$. The large difference between the observed heterozygosity $\left(\bar{H}_{o}=0.091\right)$ and gene diversity $\left(\bar{H}_{e}=0.393\right)$ and the signifi-

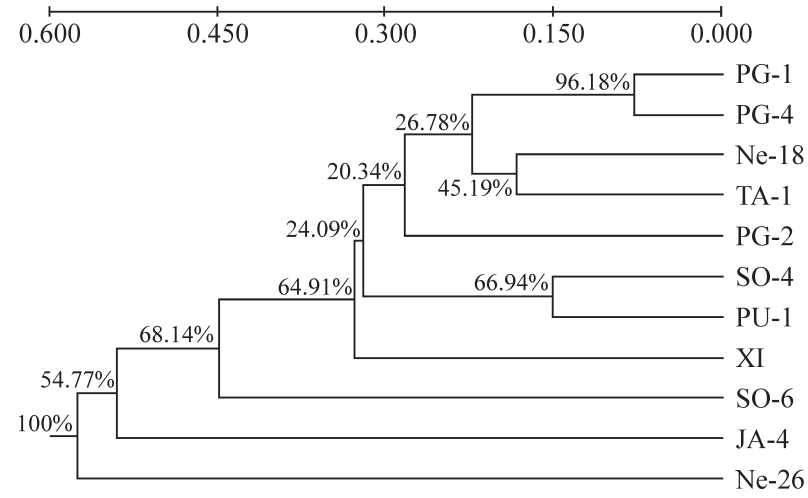

Figure 3 - Pattern of genetic divergence of 11 Brazilian Oryza glumaepatula populations based on eight microsatellite loci and unweighted pair group method with averages (UPGMA) clustering with similarities obtained from Nei's distances (1978).

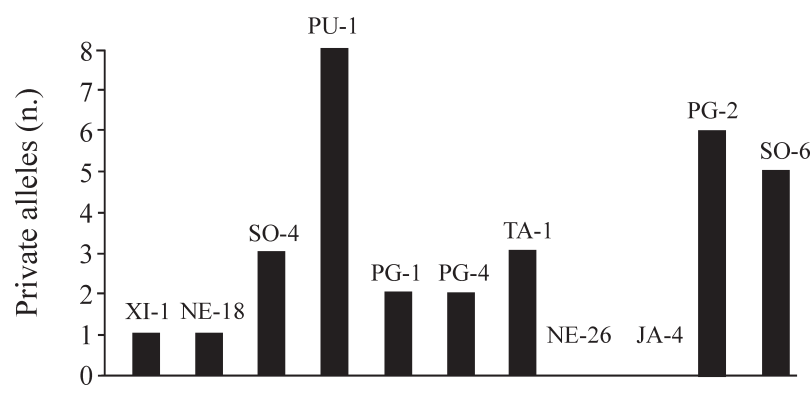

Figure 4 - Number of private alleles for the 11 Brazilian Oryza glumaepatula populations.

cance of the exact Fisher's tests for departure from HardyWeinberg proportions were caused by the prevailing mating system. These indices were superior to those reported by Buso et al. (1998) $\left(\bar{H}_{o}=0.00\right.$ to 0.025 and $\bar{H}_{e}=0.00$ to $0.21)$ and Akimoto et al. (1998) $\left(\bar{H}_{o}=0.03\right.$ and $\left.\bar{H}_{e}=0.044\right)$ with isozyme markers, probably due to the high degree of heterozygosity and higher allele number detected by the microsatellite markers in our study. However, Brondani et al. (2005) found lower values for these indices $\left(\bar{H}_{o}=0.027\right.$

Table 6 - Nei's (1978) standard genetic distances between eleven Brazilian Oryza glumaepatula populations.

\begin{tabular}{|c|c|c|c|c|c|c|c|c|c|c|c|}
\hline Populations & XI-1 & NE-18 & SO-4 & PU-1 & PG-1 & PG-4 & TA-1 & NE-26 & JA-4 & PG-2 & SO-6 \\
\hline XI-1 & $* * *$ & & & & & & & & & & \\
\hline NE-18 & 0.305 & $* * *$ & & & & & & & & & \\
\hline SO-4 & 0.309 & 0.192 & $* * *$ & & & & & & & & \\
\hline PU-1 & 0.364 & 0.363 & 0.150 & $* * *$ & & & & & & & \\
\hline PG-1 & 0.295 & 0.222 & 0.225 & 0.369 & $* * *$ & & & & & & \\
\hline PG-4 & 0.299 & 0.231 & 0.255 & 0.415 & 0.078 & $* * *$ & & & & & \\
\hline TA-1 & 0.286 & 0.184 & 0.289 & 0.450 & 0.224 & 0.206 & $* * *$ & & & & \\
\hline NE-26 & 0.650 & 0.442 & 0.527 & 0.684 & 0.445 & 0.472 & 0.436 & $* * *$ & & & \\
\hline JA-4 & 0.586 & 0.330 & 0.462 & 0.707 & 0.487 & 0.516 & 0.620 & 0.735 & $* * *$ & & \\
\hline PG-2 & 0.424 & 0.279 & 0.271 & 0.365 & 0.224 & 0.243 & 0.382 & 0.682 & 0.384 & $* * *$ & \\
\hline SO-6 & 0.450 & 0.395 & 0.475 & 0.512 & 0.425 & 0.421 & 0.450 & 0.679 & 0.760 & 0.454 & $* * *$ \\
\hline
\end{tabular}


Table 7 - List of studies on genetic diversity of several Oryza species using microsatellites (SSR) and allozyme markers, including number of loci assessed (NL), number of populations analyzed (NP), mean sample size per locus or population $(N)$, mean number of allele per locus (A), percentage of polymorphic loci $(\mathrm{p})$, gene diversity $\left(\bar{H}_{e}\right)$ and Wright's among population diversity $\left(F_{S T}\right)$ parameters.

\begin{tabular}{|c|c|c|c|c|c|c|c|c|c|}
\hline Oryza Species & Marker & $N L$ & $N P$ & $N$ & $A$ & $\mathrm{p}(\%)$ & $\bar{H}_{e}$ & $F_{S T}$ & Reference \\
\hline O. glumaepatula & SSR & 8 & 11 & $27.4^{1}$ & 3.09 & 77.3 & 0.393 & 0.491 & This study \\
\hline O. glumaepatula & Allozymes & 29 & 37 & $29.4^{2}$ & 1.18 & 16.0 & 0.044 & 0.346 & Akimoto et al. (1998) \\
\hline O. glumaepatula & Allozymes & 4 & 4 & $48.0^{2}$ & - & - & - & 0.310 & Buso et al. (1998) \\
\hline O. glumaepatula & Allozymes & 7 & 14 & $22.8^{1}$ & 1.21 & 20.7 & 0.060 & 0.763 & Veasey et al.submitted) \\
\hline O. glumaepatula & SSR & 10 & 30 & $13.8^{2}$ & 1.43 & - & 0.113 & 0.847 & Brondani et al. (2005) \\
\hline O. rufipogon & Allozymes & 22 & 21 & $28.9^{2}$ & 1.33 & 22.7 & 0.068 & 0.310 & Gao et al. (2000a) \\
\hline O. rufipogon & Allozymes & 22 & 8 & $29.1^{2}$ & 1.20 & 14.8 & 0.054 & 0.254 & Gao et al. (2002b) \\
\hline O. rufipogon & SSR & 21 & 5 & $20^{1}$ & 3.10 & 73.3 & 0.345 & 0.468 & Gao et al. (2002a) \\
\hline O. rufipogon & SSR & 10 & 12 & $19.7^{2}$ & 3.35 & 83.3 & 0.413 & 0.520 & Zhou et al. (2003) \\
\hline O. rufipogon & SSR & 6 & 47 & $26.5^{2}$ & - & - & 0.670 & 0.246 & Gao (2004) \\
\hline O. rufipogon & SSR & 21 & $4^{3}$ & $203^{2}$ & - & - & 0.609 & 0.145 & Xu et al. (2006) \\
\hline O. granulata & Allozymes & 17 & 15 & - & 1.09 & 6.3 & 0.016 & 0.859 & Gao et al. (2000b) \\
\hline O. officinalis & Allozymes & 24 & 8 & $18.1^{2}$ & 1.13 & - & 0.290 & 0.882 & Gao et al. (2001) \\
\hline O. officinalis & SSR & 14 & 18 & $24.5^{2}$ & 1.88 & 62.0 & 0.216 & 0.442 & Gao (2005) \\
\hline
\end{tabular}

${ }^{1}$ Mean sample size per locus; ${ }^{2}$ Mean sample size per population; ${ }^{3}$ Four subpopulations of a single $O$. rufipogon population.

and $\bar{H}_{e}=0.113$ ) with $O$. glumaepatula populations investigated using microsatellite markers (Table 7). The difference between our results and those of Brondani et al. could be due to both the higher number of plants per population sampled in our study (an average of 27.4 in our study compared to 13.8 by Brondani et al.) and also to the greater number of Amazon biome populations sampled by us. It should be pointed out that Brondani et al. (2005) found lower $\bar{H}_{e}$ values for populations from the Amazon biome, whilst in our study the contrary occurred in that several populations from the Amazon biome showed relatively high $\bar{H}_{e}$ values and a great heterogeneity of diversity parameters was found for these populations. Our study also provided a higher number of alleles per locus, an average of 3.09 compared to the 1.43 average found by Brondani et al. (2005). It is thus plausible to conjecture that samples larger than those used in this study could produce higher estimates for $\bar{H}_{o}, \bar{H}_{e}$ and $A$.

In microsatellite marker studies of $O$. rufipogon (Table 7) similar results were obtained by Gao et al. (2002a), who found 3.10 alleles per locus $\left(\bar{H}_{e}=0.345\right)$, and Zhou et al. (2003) who obtained 3.35 alleles per locus $\left(\bar{H}_{e}=0.413\right)$. Gao et al. (2000a, 2002b) also found lower indices in $O$. rufipogon studied with allozyme markers. The similarities between $O$. glumaepatula and O. rufipogon in terms of genetic variability contrasts with the RAPD marker results obtained by Ge et al. (1999), who reported higher genetic variability for $O$. rufipogon as compared to $O$. glumaepatula. The different results may be due to the fact that only four Amazonian O. rufipogon (O. glumaepatula) populations were evaluated by Ge et al. (1999), contrasting with the 11 populations from the Amazon biome and the
Paraguay river basin from the Pantanal biome assessed in this study and by Brondani et al. (2005), the latter study also including populations from the Cerrado biome in the Brazilian state of Goiás. In the microsatellite studies, both $O$. glumaepatula and O. rufipogon presented higher levels of genetic diversity (Table 7) when compared to $O$. officinalis Gao (2005), a wild species with a wide distribution in tropical and subtropical regions (Vaughan, 1994).

We found a high level of private alleles. Variation in the numbers of private alleles may have been due to the fact that the populations studied have undergone variable periods of genetic isolation during their evolutionary history.

\section{Mating system}

The apparent outcrossing rates estimated by us indicated different levels of cross-pollination among $O$. glumaepatula populations, varying from $2.2 \%$ to $45.9 \%$ with a mean value of $14.3 \%$. This mean value indicated a predominantly self-fertilization mating system with a small amount of outcrossing. Similar average estimates (13.5\%) were reported by Brondani et al. (2005) for $O$. glumaepatula populations.

It seems that the real distribution of outcrossing rates in O. glumaepatula is probably composed of values lower than formerly believed (Oka, 1988), indicating that the reproductive system of this species should be classified as mixed with predominant selfing. Anther and stigma lengths of the wild rice $O$. glumaepatula are generally much larger than in the cultivated rice $O$. sativa, an autogamous crop. In O. glumaepatula both anthers and stigmas are completely protruded during anthesis and generally the flowers of the same panicle do not open at the same time (Oliveira GCX, 
unpublished data), diminishing the probability of selfing by geitonogamy. It is known that $O$. glumaepatula populations occur in large flooded plains, open vegetation formations where high densities and the presence of wind provide adequate conditions for anemophily. The flowers can attract insects as well, and indeed are visited by wild bees in the Amazon under natural conditions and by domestic bees and wasps under experimental conditions (Oliveira GCX, unpublished observations), although the role of these insects as pollinators has never been assessed. The mechanism by which a large amount of selfing occurs in spite of the presence of morphological and ecological elements encouraging allogamy is still unclear, but we feel that a plausible explanation is the dehiscence of the anther accompanied by stigma receptivity before anthesis.

\section{Population genetic structure}

Our estimates of $F_{S T}(0.491)$ and $R_{S T}(0.608)$ indicated that at least $50 \%$ of the molecular diversity of $O$. glumaepatula occurred between populations, results which are not surprising for a predominantly self-fertilizing species. These relatively high values have direct consequences on the planning of conservation strategies. Considering that a given effective population size $\left(\mathrm{Ne}^{*}\right)$ is to be achieved with the sampling of $S$ populations sufficiently large to represent a metapopulation, this number must be such that $S \geq 2 F_{S T} N e^{*}$ (Vencovsky and Crossa, 2003). This inequality assumes that the actual number of populations in nature is large. With $F_{S T}=0.49$ and a desired $N e^{*}$ the result is $S \geq 0.98 \mathrm{Ne}^{*}$. Hence, many populations would then be necessary if a high $N e^{*}$ value is required.

Lower values for the $F_{S T}$ parameter for $O$. glumaepatula populations have been reported by Akimoto et al. (1998) $\left(F_{S T}=0.346\right)$ and Buso et al. (1998) $\left(F_{S T}=0.31\right)$ using allozyme markers (Table 7$)$. However, with RAPD analysis Buso et al. (1998) obtained a higher value $\left(F_{S T}=0.64\right)$, similar to the $R_{S T}$ values. Also, Brondani et al. (2005) reported that population subdivision was responsible for the major increase in inbreeding for this species when using microsatellite markers, with values of $F_{S T}=0.85, R_{S T}=0.90, F_{I S}=0.79$ and $F_{I T}=0.98$. The higher $F_{S T}$ values found with microsatellite markers by Brondani et al. (2005) may be explained by the smaller number (6 to 24) of plants sampled to represent the population, which may have overestimated the divergence between populations. A parallel study of O. glumaepatula (Silva CM, Karasawa MMG, Veasey EA, unpublished data) with a small sample (12 to 24 plants) per population also provided higher $\mathrm{F}$ statistic values $\left(F_{I T}=0.963 ; F_{I S}=0.858\right.$; $F_{S T}=0.715$ ), although a similar result with allozyme data was obtained when evaluating 14 O. glumaepatula populations (Veasey EA, Cardin DC, Silva RM, Bressan EA, Vencovsky R, submitted) with seven loci and an average of 22.8 sampled plants per locus. With allozyme markers, $O$. officinalis (Gao et al., 2001) and O. granulata (Gao et al., 2000b) have also shown higher levels of population differentiation (Table 7), although lower levels were detected for $O$. officinalis when assessed with microsatellite markers (Gao, 2005), similar to the values observed in our study. Gao (2005) has suggested that the different results achieved when using allozyme and microsatellite markers in $O$. officinalis are due to differences in interpopulation distances.

When studying the genetic diversity among $O$. rufipogon populations from Brazil (O. glumaepatula) and China, Ge et al. (1999) reported that genetic diversity between populations differed between these continents. Molecular variance analysis (AMOVA) with RAPD data showed that the percentage of genetic diversity between populations was $52.74 \%$ for the Brazilian and $28.75 \%$ for the Chinese populations. Even lower variability between populations was found in O. rufipogon using microsatellite markers, when examining Chinese populations and subpopulations (Gao, 2004; Xu et al., 2006). Higher diversity levels and higher within population variability found in Chinese O. rufipogon populations, in relation to South American $O$. glumaepatula populations, have been attributed to the higher outcrossing rates observed in Chinese populations (Ge et al., 1999).

In our Brazilian O. glumaepatula populations the large genetic differentiation between populations was, as discussed above, a reflection of the predominantly self-fertilization mating system which reduced gene flow between populations. The mating system and the consequently high intrapopulation inbreeding $\left(F_{I S}=0.78\right)$ was also the major factor responsible for the high total inbreeding $\left(F_{I T}=0.888\right)$ detected in our study. Other reports on $O$. glumaepatula reproductive system have also been associated with high values of total inbreeding, such as Akimoto et al. (1998) having reported $F_{I S}=0.769, F_{I T}=0.965$ and $F_{S T}=0.346$ and Buso et al. (1998) $F_{I S}=0.930, F_{I T}=0.970$ and $F_{S T}=0.310$.

In our study, low values were estimated for the number of migrants per generation $(\mathrm{Nm}=0.259$ and 0.161 for $F_{S T}$ and $R_{S T}$, respectively). Gene flow estimated by the $R_{S T}$ parameter was lower and is probably a more precise measure since $R_{S T}$ provides less biased estimates of microsatellite demographic parameters for a population than $F_{S T}$ (Slatkin, 1995). $N m$ values are related to the $F_{S T}$ or $R_{S T}$ parameters, according to the equation $N m=1 / 4(1 / R s t-1)$, whereas the $R_{S T}$ depends on the capacity of the marker to show polymorphism and the capacity of the sampled plants to represent the total variability existing in the population. When the within population diversity is low, the effect of the subdivision tends to be pronounced because the total diversity in the species is found between populations.

The low gene flow values obtained in our study are not in accordance with those found by Buso et al. (1998) who observed higher gene flow $(\mathrm{Nm}=0.73)$ among four $O$. glumaepatula populations, probably because, as mentioned above, a low polymorphism marker (allozymes) was used 
in that study. Buso et al. (1998) classified gene flow as intermediate, according to the three levels of gene flow for highly autogamic species described by Govindajaru (1989) based on $\mathrm{Nm}$ estimations: high, $\mathrm{Nm}>1$; intermediate, $0.250<N m<0.999$; and low, $N m<0.249$ ). Gao (2004) also found limited gene flow among Chinese $O$. rufipogon populations $(\mathrm{Nm}=0.766)$. Akimoto et al. $(1998)$ have emphasized that gene flow in Amazonian O. glumaepatula populations seems to be due to seed dispersal rather than pollen dispersal, and that gene flow proceeds mainly in the direction of river flow. In fact, the viability of pollen grains in the genus Oryza is, in general, limited to a few minutes (Oka, 1988), which makes gene flow between populations separated by a few hundred meters impossible. Even in regions with the highest densities, populations are separated by rivers, tributaries, lakes, channels and forests that are usually many hundreds of meters wide. Thus, pollen migration must play a minor role in gene flow. On the other hand, zoochory (see below) and dispersal of whole plants by programmed abscission followed by floating downstream must be the factors responsible for the $\mathrm{Nm}$ values observed.

In our study, all 11 O. glumaepatula populations formed random groupings in the cluster analysis and not according to their geographic origin. Hence, the hypothesis previously formulated that the more geographically distant populations would also be genetically more differentiated was discarded by the results of the correlation analysis, which showed no correlation between the genetic and geographic distances $(r=0.03)$. This result indicates that geographic proximity is not indicative of genetic similarity and, therefore, is not a guide for understanding the genetic structure of this species. On the other hand, comparable cluster analyses based on allozyme markers have placed $O$. glumaepatula populations from the Brazilian Pantanal Matogrossense and those originated in the Amazon region in different groups (Veasey EA, Cardin DC, Silva RM, Bressan EA, Vencovsky R, submitted). This result may be due to a selection effect that may appear in allozyme markers, resulting from the expression of adaptive genes (Ferreira and Grattapaglia, 1998) as it was evident that certain alleles were mostly predominant in the Amazon biome populations whereas other alleles of the same locus were predominant in the Pantanal biome populations. However, this was not observed with microsatellite markers because these markers are not adaptive and hence the genetic drift effect is random throughout evolutionary time (Pemberton et al., 2000).

The geographic distribution of the genetic variation in O. glumaepatula is not expected to be simple to describe or explain from either an historical or adaptive point of view. The reason for this is the apparently intense migration between and within basins, following unpredictable timing and direction. No systematic study on migration and dispersal of Amazonian wild rice has ever been performed, in part because of its inherent difficulty, but a few reports from various sources partly reveal the complexity of the situation. The principal habitats of $O$. glumaepatula are the flooded grasslands and flooded forests on the Quaternary terrains found on the banks of the major rivers of the Amazonian Basin and Pantanal. These terrains are not homogeneously distributed along the rivers but instead are concentrated in patches, mainly on the left banks of the Middle Amazon, the confluence of the Japurá with the Solimões rivers, the confluence of the Purus with the Solimões, the headwaters of the River Xingu, the Bananal Island in the Araguaia, the Guaporé Basin, the central north coast of the Brazilian state of Amapá, the Paraguay basin and the plains around the River Negro. These are the regions where most of the O. glumaepatula populations occur, although sparse and less dense populations are spread among them.

The river banks and flooded plains which are the main habitat of members of the genus Oryza can be eroded and quickly become uncolonizable. Satellite image analyses have revealed that the fusiform islands typical of the Solimões/Amazon, which are rich in wild rice populations, normally shift along the river as a result of erosion-deposition. Such geographical mobility may be the cause of rapid cycles of population extinction and colonization, explaining part of the lack of significance of the correlation coefficient and the absence of clustering of geographically contiguous river basins. This also makes the delimitation of priority areas for in situ conservation more difficult. Also, the multiple agents and directions of the dispersal movements among the river basins in the Amazon (Morishima and Martins, 1994) permit the insertion of recently established, unrelated, long-distance immigrant populations, and may partially explain the loose geographical structure so far observed.

\section{Conservation Remarks}

This study reveals the existence of diversity both within and between river basins, as well as of alleles particular to each population. Studies like these can be useful in setting guidelines both for in situ conservation and for collecting and maintaining germplasm in banks, having in mind short-term conservation. For instance, the mean number of alleles enables the populations to be put in order of genetic richness, and to conclude, at least under the current state of knowledge, that populations PG-4 and PG-2, in the Paraguay River system, and PU-1, in the Amazon, are conservation priorities. High genetic diversity is also another criteria to be taken into consideration. Therefore, populations SO-4 and NE-18, from the Amazon, should also be considered as conservation priorities. In addition, both $F_{S T}$ and $\bar{t}_{a}$ indicate that, in most cases, it is advisable to sample many populations. The high level of genetic differentiation found between populations and the high number of private alleles indicate the necessity of sampling a large number of populations for ex situ conservation. The same is true for in 
situ conservation, but long-term in situ conservation demands more studies over long periods on the dynamics of the populations. Allelic richness was found in our study even for populations isolated from cultivated materials, in contrast with conclusions drawn previously (Brondani et al., 2005).

Based on data obtained in this study and in Brondani et al. (2005) and Silva CM, Karasawa MMG, Veasey EA (unpublished data), we suggested that future studies should not only increase the number of populations collected, but also the number of plants sampled per population. This procedure will provide a better estimate of the real allelic diversity in the populations, as these estimates will have a direct effect on the values obtained for other diversity and population genetic structure estimates.

A further study suggested is the possible effects of the breeding system and genetic drift over generations in $O$. glumaepatula populations, which could be carried out using computer simulations. A preliminary study investigated the effect of the breeding system on the structure and genetic diversity within and between populations (XI-1, SO-6 and PG-1) for one generation. Results indicated high fixation indices within each family on each population but the total within population diversity was maintained after a generation (Karasawa MMG, Vencovsky, R., Zucchi, MI, Oliveira, GCX, Veasey, EA, unpublished data). Based on this preliminary study we suggest further studies to determine the real evolutionary effect of the reproductive system on the distribution of genetic diversity over many generations, to establishing the most effective conservation practices for O. glumaepatula species.

\section{Acknowledgments}

The authors wish to thank the Brazilian research councils CNPq and FAPESP and the Ministry of Education of Japan for grants and financial supports, Dr. Elaine Cristina Cardoso Fidalgo for help with the satellite images, the National Institute of Amazonian Research (INPA, Manaus, Brazil) for collaboration during field collecting activities, and the National Institute of Space Research (INPE, Brazil). The authors are also grateful to two anonymous reviewers for valuable comments on the manuscript.

\section{References}

Akimoto M, Shimamoto Y and Morishima H (1998) Population genetic structure of wild Oryza glumaepatula distributed in the Amazon flood area influenced by its life-history traits. Mol Ecol 7:1371-1381.

Bassam BJ, Caetano-Anolles G and Gresshoff PM (1991) Fast and sensitive silver staining of DNA in polyacrylamide gels. Anal Biochem 196:80.

Bautista NS, Solis R, Kamijima O and Ishii T (2001) RAPD, RFLP and SSLP analyses of phylogenetic relationships between cultivated and wild species of rice. Genes Genet Syst 76:71-79.
Brondani C, Brondani RPV, Rangel PHN and Ferreira ME (2001) Development and mapping of Oryza glumaepatula-derived microsatellite markers in the interspecific cross Oryza glumaepatula x O. sativa. Hereditas 134:59-71.

Brondani RPV, Zucchi MI, Brondani C, Rangel PHN, Borba TCO, Rangel PN, Magalhães MR and Vencovsky R (2005) Genetic structure of wild rice Oryza glumaepatula populations in three Brazilian biomes using microsatellite markers. Genetica 125:115-123.

Buso GSC, Rangel PH and Ferreira ME (1998) Analysis of genetic variability of South American wild rice populations (Oryza glumaepatula) with isozymes and RAPD markers. Mol Ecol 7:107-117.

Buso GSC, Rangel PH and Ferreira ME (2001) Analysis of random and specific sequences of nuclear and cytoplasmatic DNA in diploid and tetraploid American wild rice species (Oryza spp.). Genome 44:476-494.

Doi K, Yoshimura A, Nakano M and Iwata N (1996) Classification of A genome species in the genus Oryza using nuclear DNA markers. Int Rice Res Notes 21:2-3.

Doi K, Nomura MN, Yoshimura A, Iwata N and Vaughan DA (2000) RFLP relationships of A-genome species in the genus Oryza. J Fac Agr Kyushu U 45:83-98.

Ferreira ME and Grattapaglia D (1998) Introdução ao Uso de Marcadores Moleculares em Análise Genética. 3rd edition. Embrapa/Cenargen, Brasília, 220 pp.

Gao LZ (2004) Population structure and conservation genetics of wild rice Oryza rufipogon (Poaceae): A region-wide perspective from microsatellite variation. Mol Ecol 13:10091024.

Gao LZ (2005) Microsatellite variation within and among populations of Oryza officinalis (Poaceae), an endangered wild rice from China. Mol Ecol 14:4287-4297.

Gao LZ, Ge S and Hong DY (2000a) Allozyme variation and population genetic structure of common wild rice Oryza rufipogon Griff. in China. Theor Appl Genet 101:494-502.

Gao LZ, Ge S and Hong DY (2000b) Low levels of genetic diversity within population and high differentiation among populations of a wild rice, Oryza granulata Nees et. Arn. ex. Watt. from China. Int J Plant Sci 161:691-697.

Gao LZ, Ge S and Hong DY (2001) High levels of genetic differentiation of Oryza officinalis Wall. et Watt. from China. J Hered 92:511-516.

Gao LZ, Schaal BA, Zhang CH, Jia JZ and Dong YS (2002a) Assessment of population genetic structure in common wild rice Oryza rufipogon Griff. using microsatellite and allozyme markers. Theor Appl Genet 106:173-180.

Gao LZ, Ge S and Hong DY (2002b) Allozyme variation and conservation genetics of common wild rice (Oryza rufipogon Griff.) in Yunnan, China. Euphytica 124:273-281.

Gao LZ, Zhang CH, Chang LP, Jia JZ, Qiu ZE and Dong YS (2005) Microsatellite diversity within Oryza sativa with emphasis on indica-japonica divergence. Genet Res 85:1-14.

Ge S, Oliveira GCX, Schaal BA, Gao LZ and Hong DY (1999) RAPD variation within and between natural populations of the wild rice Oryza rufipogon from China and Brazil. Heredity $82: 1-7$.

Goodman SJ (1997) $\mathrm{R}_{\mathrm{ST}}$ Calc: A collection of computer program for calculating estimates of genetic differentiation from microsatellite and determining their significance. Mol Ecol 6:881-885. 
Govindajaru RD (1989) Variation in gene flow levels among predominantly self-pollinated plants. J Evolution Biol 2:173181.

Hedrick PW (2001) Conservation genetics: Where are we now? Trends Ecol Evol 16:629-636.

Hoisington D, Khairallah M and Gonzalez-de-Leon D (1994) Laboratory Protocols: CYMMYT Applied Molecular Genetics Laboratory. CYMMYT, Mexico, 88 pp.

Holsinger KE (2000) Reproductive systems and evolution in vascular plants. P Natl Acad Sci USA 97:7037-7042.

Karasawa MMG (2005) Análise da estrutura genética de populações e sistema reprodutivo de Oryza glumaepatula por meio de microssatélites. Ph.D. Thesis, Escola Superior de Agricultura "Luiz de Queiroz", Universidade de São Paulo, Piracicaba.

Lewis PO and Zaykin D (2000) Genetic Data Analysis: Computer Program for the Analysis of Allelic Data. Version 1.0 (d15). http://alleyn.eeb.uconn.edu/gda.

Mantel N (1967) The detection of disease clustering and a generalized regression approach. Cancer Res 27:209-220.

Miller M (1997) Tools For Population Genetic Analyses (TFPGA) 1.3: A Windows Program for Analysis of Allozyme and Molecular Population Genetic Data. http://www. public.asu.edu.

Morishima H and Martins PS (1994) Investigations of plant genetic resources in the Amazon Basin with emphasis on the genus Oryza. Report of the 1992/93 Amazon Project. The Monbusho International Scientific Research Program, Japan, and FAPESP, Brazil, 100 pp.

Nei M (1978) Estimation of average heterozygosity and genetic distance from a small number of individuals. Genetics 89:583-590.

Oka HI (1988) Origin of Cultivated Rice. Japan Scientific Societies Press, Tokyo, and Elsevier Science Publishers, Amsterdam, $254 \mathrm{pp}$.

Oliveira GCX (1994) Geographic distribution of wild Oryza species in Brazil. In: Morishima $\mathrm{H}$ and Martins PS (eds) Investigations of Plant Genetic Resources in the Amazon Basin with Emphasis on the Genus Oryza. Report of 1992/93 Am- azon Project, The Monbusho International Scientific Research Program, Japan, and FAPESP, Brazil, pp 10-15.

Oliveira GCX (2002) A molecular phylogenetic analysis of Oryza L. based on chloroplast DNA sequences. Ph.D. Thesis, Washington University, St. Louis.

Peatkau D, Calvert W, Stirling I and Strobeck C (1995) Microsatellite analysis of population structure in Canadian Polar bears. Mol Ecol 4:347-354.

Pemberton JM, Coltman C, Coulson TN and Slade J (2000) Using microsatellites to measure the fitness consequence of inbreeding and outbreeding. In: Goldstein DB and Schlötterer C (eds) Microsatellites: Evolution and Applications. Oxford University Press, New York, pp 151-164.

Richards AJ (1997) Plant Breeding Systems. 2nd edition. Chapman and Hall, London, 529 pp.

Rohlf J (1992) NTSYS-pc: Numerical Taxonomy and Multivariate Analysis System. Version 1.70 (software). Stony Brook, New York.

Slatkin M (1995) A measure of population subdivision based on microsatellite allele frequencies. Genetics 139:457-462.

Vaughan DA (1994) The Wild Relatives of Rice: A Genetic Resources Handbook. International Rice Research Institute, Manila, $137 \mathrm{pp}$

Vaughan DA, Morishima H and Kadowaki K (2003) Diversity in the Oryza genus. Curr Opin Plant Biol 6:139-146.

Vencovsky R and Crossa J (2003) Measurements of representativeness used in genetic resources conservation and plant breeding. Crop Sci 43:1912-1921.

Weir BS (1996) Genetic Data Analysis II: Methods for Discrete Population Genetic Data. Sinauer Associates, Inc. Publishers, Sunderland, $445 \mathrm{pp}$.

Xu X, Lu BR, Chen YH, Xu M, Rong J, Ye P, Chen J and Song Z (2006) Inferring population history from fine-scale spatial genetic analysis in Oryza rufipogon (Poaceae). Mol Ecol 15:1535-1544.

Zhou HF, Zhong WX and Ge S (2003) Microsatellite analysis of genetic diversity and population genetic structure of a wild rice (Oryza rufipogon Griff.) in China. Theor Appl Genet 107:332-339

Associate Editor: Márcio de Castro Silva Filho 